\title{
Electric Field Induced Spectra of Molecular Hydrogen, Deuterium and Deuferium Hydride
}

\author{
P. J. Brannon \\ Department of Physics, The University of Tennessee, Knoxville, Tennessee 37916
}

C. H. Church

Westinghouse Research Laboratories, Pittsburgh, Pennsylvania 15285

AND

C. W. PETERS

Department of Physics, University of Michigan, Ann Arbor, Michigan 48104

\begin{abstract}
The electric field induced spectra of molecular hydrogen, deuterium and deuterium hydride was observed in the pressure range of 0 to $600 \mathrm{psi}$. Thirteen lines each of the fundamental vibration-rotation band were observed for hydrogen and deuterium and nine were observed for deuterium hydride. A plot of line position versus density yielded zero pressure line positions. Molecular constants were derived from the zero pressure values.
\end{abstract}

\section{INTRODUCTION}

The fundamental vibration-rotation energies of the hydrogen molecule may be determined by the spectroscopic examination of Raman scattering, electric quadrupole absorption or electric field induced absorption. Ordinary electric dipole absorption is ruled out by the symmetry of the molecule. These processes are about a million times weaker than the ordinary dipole absorption, and hence, a larger sample is needed to give an observable effect. The increase can be obtained by longer path lengths of the gas, higher pressures or both.

An appreciable increase of the pressure of a gas usually leads to shifts and broadening of the spectral lines. These effects are not nearly as great in hydrogen as they are in an ordinary polar gas, but they are observable at pressures of a few atmospheres. Since the spectroscopic processes mentioned above require pressures of a few atmospheres or greater, it is necessary in all three cases to extrapolate the data of the spectral line positions to zero pressures in order to determine accurate molecular constants.

May et al. (1), (2) observed the pressure dependence of the Raman spectrum 
of hydrogen for the pressure range of 100 to 2000 atmospheres. They were able to fit their data for each line to the sum of a linear and quadratic term in the density. The linear term gave rise to a negative shift in frequency and was predominant for pressures less than 100 atmospheres. The quadratic term gave rise to a positive shift, and became predominant for pressures greater than 400 atmospheres. They also found the shifts varied slightly for different $J$ transitions. More recently Rank et al. (3)-(5) extrapolated electric quadrupole, Raman and electric field induced data to zero.

The other and more common aspect of the effect of pressure on the observed spectrum is line broadening due to collisions between molecules. May et al. (1), (2) reported approximate values for the line widths in their high pressure Raman studies that indicated the broadening was linearly proportional to the density and varied only slightly for different transitions in the vibration-rotation fundamental band. Allin et al. $(6)$ found that the line widths varied linearly with density in the range 30 to 600 atmospheres. Rank et al. (5) also found that a linear dependence of the width of $Q_{1}(1)$ line on density. A theoretical treatment of the problem of line broadening for the Raman spectrum of hydrogen was given for low pressures by Fiutak and Van Kranendonk $(\boldsymbol{\gamma})$. Their theory was basically an Andersontype theory with quadrupoles interacting. Because the pertinent matrix elements are the same for Raman scattering and electric field induced absorption, their calculations can also be applied to the latter. Van Kranendonk $(8)$ predicted the half widths of the $Q$ and $S$ lines for $J$ from 0 through 4 .

As mentoned above, the vibration-rotation spectrum of hydrogen can be determined by different methods: Raman scattering, quadrupole absorption, and electric field induced absorption. Electric ficld induced absorption was chosen in this study.

Condon first gave the theory of electric field induced spectra in $1932(9)$. However, because of technological difficulties, it was not observed until 1953 by Craw ford and Dagg (10), in 1958 by (rawford and MacDonald (11), and in 1959 by Terhune and Peters (12). Hydrogen was the molecule studied in all cases because its fundamental vibration-rotation band falls in a spectral region where sensitive detectors are available. Crawford et al. (10), (11) used a de electric field, whereas, Terhume and Peters (12) used a low-frequency alternating electric field to modulate the absorption. Rank et al. (5) used a combination of both.

\section{EXPERIMENTAL EQUIPMENT AND TECHNIQUES}

A block diagram of the experimental arrangement is shown in Fig. 1. The spectrometer was a three meter Ebert type which is described in detail by Marshall (13). Type $\mathrm{O}$ and $\mathrm{P}$, coolable, Eastman Kodak, lead sulfide detectors were used. The $\mathrm{P}$ ' type was used at the longer wavelengths. The sources were a carbon arc lamp and a quartz iodine tungsten filament lamp. The carbon arc was used in all regions where $\mathrm{CO}_{2}$ and $\mathrm{H}_{2} \mathrm{O}$ did not interfere.

The sample cell consisted of two steel plates, ground flat and chrome plated, 


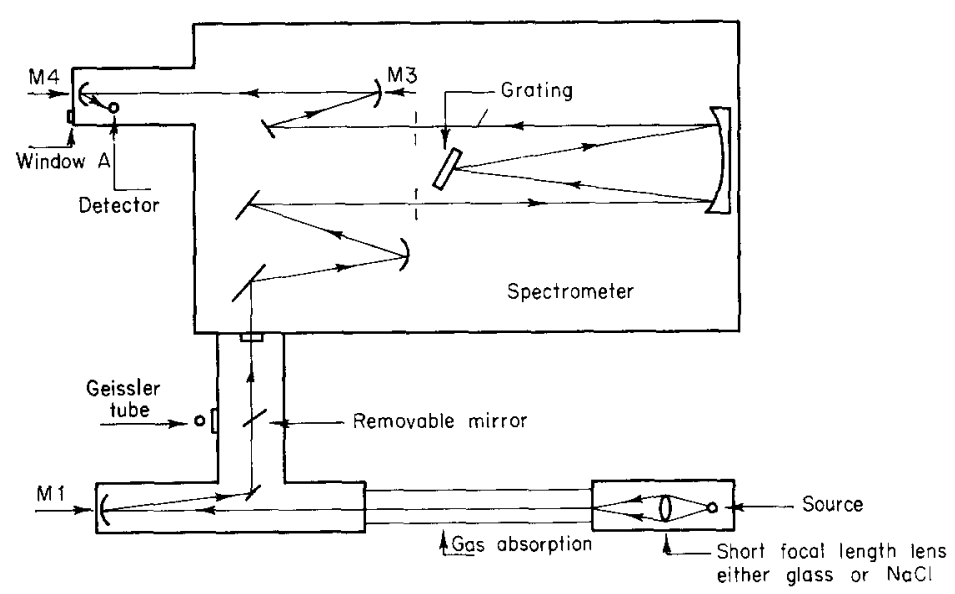

Fig. 1. Block Diagram of Spectrometer and Optics.

enclosed in a thick-walled pipe. The steel plates were separated by plastic spacers, $2 \mathrm{~mm}$ thick, placed along the long edge. The plates and spacers were cemented together with Araldite epoxy resin. The dimensions of the steel plates were $4 \times 90$ $\mathrm{cm}$ by $1 / 2$ in. thick. The end caps of the thick-walled pipe had $\mathrm{CaF}_{2}$ windows. The steel plates acted as a light guide for the infrared radiation. The cell was operated at $f-30$ and a $20 \%$ transmission coefficient was measured for the cell at $2.5 \mu$. Lucite blocks were machined to fill the excess space so as to reduce the volume to a minimum.

The voltage was applied to the steel plates as dc or a square wave. The high voltage necessary for the experiment was supplied by a rf type de supply similar to that described by Mautner and Schade (14). For the static electric field measurements, this supply was connected directly to the cell. In the case of the modulated field technique, a rotating spark gap was used to connect the cell to the high voltage and then to ground at a $90 \mathrm{cps}$ rate. 'The dc method was used whenever possible, since higher fields could be obtained without the gas breaking down.

The rotating spark gap consisted of a plexiglas wheel with three magnesium electrodes spaced $120^{\circ}$ apart, all connected to a center electrode which led to the gas cell. As the wheel rotated at $1800 \mathrm{rpm}$, it swept the three electrodes past two fixed electrodes $180^{\circ}$ apart, one connected to the high-voltage supply, the other to ground.

The high voltage signal was measured through a resistor potential divider whose ratio was 2026 to 1 . The ratio was measured with a Rubicon type-B potentiometer. The voltage across the smaller resistor in the divider was measured by two methods. In the modulated operation, an oscilloscope was used to measure the peak-to-peak voltage of the square wave. In the dc operation, the voltage across the small resistor was measured by a high resistance voltmeter. 
The lines were calibrated with atomic emission lines of $\mathrm{Ar}, \mathrm{Kr}, \mathrm{Xe}, \mathrm{He}$ and $\mathrm{Ne}$ in higher orders. In every case, two emission lines were used to calibrate the infrared line. In most cascs, the calibration lines werc less than $15 \mathrm{~cm}^{-1}$ apart. A linear extrapolation was made from the emission lines to the infrared line. In the cases where dispersion effects were appreciable, the appropriate corrections were made. The wavelengths of most of the emission lines used were known to within a few thousandths of a wave number. There were, however, a few lines which had errors of order $0.02 \mathrm{~cm}^{-1}$; most of these were used in calibration $\mathrm{D}_{2}$. The conversion from air wavelengths to vacuum wave numbers was made with the aid of $\mathrm{Na}$ tional Bureau of Standards tables (Monograph 3 ).

The beam from the emission lamp was introduced into the spectrometer by means of a removable diagonal mirror (See Fig. 1). The emission beam followed the same path as the infrared beam. The emission lamp was located just outside the chopper housing in a position such that it would be focused on the entrance slit without any additional optical elements other than the diagonal mirror. A special holder was constructed so that the emission lamps could be changed readily.

The hydrogen gas used in this work was purchased from The Matheson Company and was their ultra-pure grade. The deuterium gas was purchased from General Dynamics Iiquid Carbonic Company. Its purity was $99.7 \%$. The deuterium hydride gas was obtained from Merck, Sharp, and Dohme. Its purity was $97 \%$.

The zero pressure line positions were determined by best fitting position versus density and extrapolating to zero. For the range of pressure in this experiment ( 0 to 600 psi), it was assumed a linear relation existed between line position and density. This was based on the data of May et al. (1), (2). The errors indicated in Table $\mathrm{X}$ are the statistical errors given by the best fit. The line position errors take into consideration the error determined by measuring a known line, the strength of the line, and the statistical errors in Table X. Molecular constants were derived from Dunham's (15) expression for the energy.

where $T(V, J)=G(V)+F_{u}(J)$,

$$
\begin{aligned}
F_{v}(J) & =B_{v} J(J+1)-D_{v} J^{2}(J+1)^{2}+H_{v} J^{3}(J+1)^{3}, \\
v & =\text { vibrational quantum number, } \\
J & =\text { rotational quantum number, }
\end{aligned}
$$

and $G(V)$ depends only upon the vibrational quantum number. The errors on the quoted molecular constants are statistical.

Most of the lines of hydrogen were observed with slit widths of 0.15 to 0.20 $\mathrm{cm}^{-1}$. For deuterium, they varied from 0.16 to $0.25 \mathrm{~cm}^{-1}$. Because of the limited supply of deuterium hydride, the spectral slit widths were $0.3 \mathrm{~cm}^{-1}$ in most cases. Also the spectra was observed at one pressure only (100 psi) and was not extrapoated to zero as was deuterium and hydrogen. The overtone lines of hydrogen and deuterium were taken with $0.40 \mathrm{~cm}^{-1}$ slits. Typical data is shown in Figs. 2 and 3. 

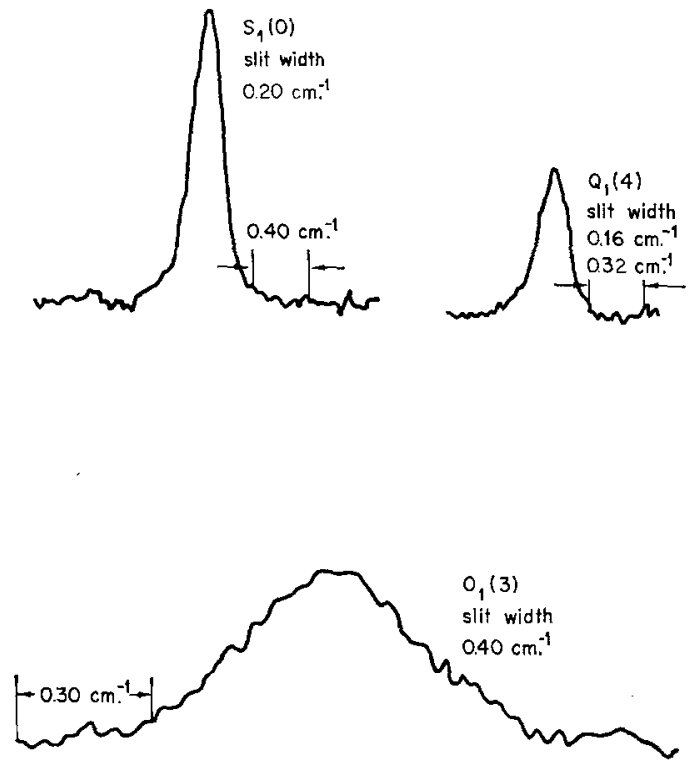

Fig. 2. $S_{1}(0), O_{1}(3)$ and $Q_{1}(4)$ of $\mathrm{H}_{2} .121,000 \mathrm{~V} / \mathrm{cm}, 400$-psi, 4-sec response for $S_{1}(0)$ and $Q_{1}(4), 12-$ sec response for $O_{1}(3)$.
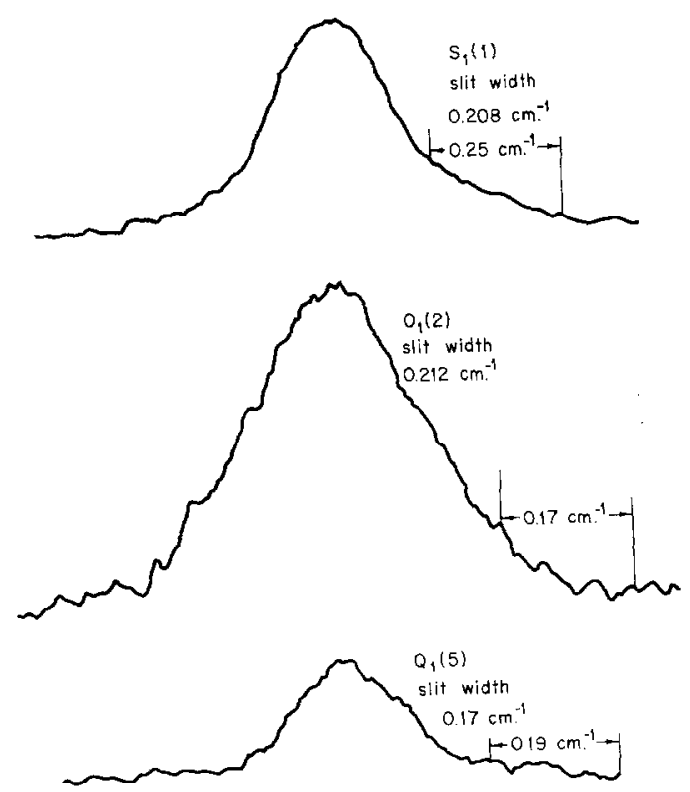

FIG. 3. $S_{1}(1), O_{1}(2)$ and $Q_{1}(5)$ of $D_{2} .121,000 \mathrm{~V} / \mathrm{cm}, 400$-psi, 12-sec response. 
TABLE 1

Obsarved and Calculated Spectrum of $\mathrm{D}_{2}$

\begin{tabular}{cccc}
\hline Line & Obs. Value & Calc. Value & Obs. Calc. \\
\hline$Q_{1}(0)$ & $2993.600 \pm 0.02 \mathrm{~cm}^{-1}$ & $2993.602 \mathrm{~cm}^{-1}$ & -0.002 \\
$Q_{1}(1)$ & $2991.486 \pm 0.02$ & 2991.489 & -0.003 \\
$Q_{1}(2)$ & $2987.268 \pm 0.02$ & 2987.272 & -0.004 \\
$Q_{1}(3)$ & $2980.967 \pm 0.02$ & 2980.968 & -0.001 \\
$Q_{1}(4)$ & $2972.597 \pm 0.03$ & 2972.597 & 0.000 \\
$Q_{1}(5)$ & $2962.176 \pm 0.04$ & 2962.170 & 0.006 \\
$Q_{1}(6)$ & $2949.685 \pm 0.04$ & 2949.669 & 0.016 \\
$S_{1}(0)$ & $3166.300 \pm 0.02$ & 3166.301 & -0.001 \\
$S_{1}(1)$ & $3278.434 \pm 0.02$ & 3278.448 & -0.014 \\
$S_{1}(2)$ & $3387.220 \pm 0.02$ & 3387.193 & 0.027 \\
$S_{1}(3)$ & $3492.064 \pm 0.03$ & 3492.052 & 0.012 \\
$S_{1}(\mathbf{4})$ & & 3592.559 & \\
$O_{1}(2)$ & $2814.530 \pm 0.04$ & 2814.573 & 0.043 \\
$O_{1}(3)$ & $2694.050 \pm 0.04$ & 2694.008 & 0.042 \\
$Q_{2}(0)^{\mathrm{a}}$ & $5868.00 \pm 0.08$ & & \\
$Q_{2}(1)^{\mathrm{a}}$ & $5863.84 \pm 0.08$ & & \\
$Q_{2}(2)^{\mathrm{a}}$ & $5855.48 \pm 0.08$ & & \\
\hline
\end{tabular}

at 530 psi.

TABLE II

Observed and Calculated $\mathrm{H}_{2}$ Spectrum

\begin{tabular}{cccr}
\hline Line & Obs. Value & Calc. Value & Obs. Calc. \\
\hline$Q_{1}(0)$ & $4161.158 \pm 0.02 \mathrm{~cm}^{-1}$ & $4161.158 \mathrm{~cm}^{-1}$ & 0.000 \\
$Q_{1}(1)$ & $4155.240 \pm 0.02$ & 4155.240 & 0.000 \\
$Q_{1}(2)$ & $4143.449 \pm 0.02$ & 4143.449 & 0.000 \\
$Q_{1}(3)$ & $4125.867 \pm 0.02$ & 4125.866 & 0.001 \\
$Q_{1}(4)$ & $4102.595 \pm 0.03$ & 4102.596 & -0.001 \\
$Q_{1}(5)$ & $4073.745 \pm 0.05$ & 4073.745 & 0.000 \\
& & & \\
$S_{1}(0)$ & $4497.807 \pm 0.02$ & 4497.820 & -0.013 \\
$S_{1}(1)$ & $4712.923 \pm 0.02$ & 4712.889 & 0.034 \\
$S_{1}(2)$ & $4916.970 \pm 0.03$ & 4916.997 & -0.027 \\
$S_{1}(3)$ & $5108.386 \pm 0.04$ & 5108.367 & 0.019 \\
& & & \\
$O_{1}(2)$ & $3806.780 \pm 0.04$ & 3806.788 & 0.008 \\
$O_{1}(3)$ & $3568.222 \pm 0.04$ & 3568.217 & 0.005 \\
$O_{1}(4)$ & $3329.050 \pm 0.04$ & 3329.049 & 0.001 \\
& & & \\
$Q_{2}(0)^{\mathrm{a}}$ & $8086.86 \pm 0.08$ & & \\
$Q_{2}(1)^{\mathrm{a}}$ & $8075.27 \pm 0.04$ & & \\
$Q_{2}(2)^{\mathrm{s}}$ & $8051.80 \pm 0.08$ & & \\
\hline
\end{tabular}

a $550 \mathrm{psi}$. 


\section{RESULTS}

The observed zero pressure line positions along with the calculated ones are listed in Tables I-III. The calculated ones are based on the best fit to the observed data. In the case of deuterium hydride, $\mathrm{H}_{0}$ and $\mathrm{H}_{1}$ were calculated from an expression given by Dunham (15), and the values of these constants for hydrogen and deuterium. 'Iables III-V compare the data of this experiment with that found by others.

The molecular constants of this experiment are compared to those of others in Tables VI-VIII. It is noted that the constants for hydrogen and deuterium are within experimental error of those found by Rank et al. (5) and Stoicheff (16).

TABLE III

Orserved and Calculated Spectrum of HD of This Experiment Compared to Otiens

\begin{tabular}{clll}
\hline Line & \multicolumn{1}{c}{ Obs. This Exp. } & Calc. This Exp. & \multicolumn{1}{c}{ Stoicheff } \\
\hline$Q_{1}(0)$ & $3632.165 \pm 0.03 \mathrm{~cm}^{-1}$ & $3632.160 \mathrm{~cm}^{-1}$ & $3632.055 \mathrm{~cm}^{-1}$ \\
$Q_{1}(1)$ & $3628.278 \pm 0.03$ & 3628.300 & 3628.197 \\
$Q_{1}(2)$ & $3620.616 \pm 0.03$ & 3620.603 & 3620.511 \\
$Q_{1}(3)$ & $3609.093 \pm 0.03$ & 3609.114 & 3608.992 \\
$Q_{1}(4)$ & $3593.910 \pm 0.04$ & 3593.893 & \\
$S_{1}(0)$ & $3887.668 \pm 0.03$ & 3887.692 & \\
$S_{1}(1)$ & $4052.214 \pm 0.03$ & 4052.182 & \\
$S_{1}(2)$ & $4209.870 \pm 0.03$ & 4209.880 & \\
$O_{1}(2)$ & $3365.056 \pm 0.05$ & 3365.081 & \\
\hline
\end{tabular}

TABLE IV

A Comparison of $D_{2}$ Spectrum of This Experiment with That Observed by Others

\begin{tabular}{clc}
\hline Line & Obs. in This Exp. & Stoicheff RamanEffect \\
\hline$Q_{1}(0)$ & $2993.600 \mathrm{~cm}^{-1}$ & $2993.548 \mathrm{~cm}^{-1}$ \\
$Q_{1}(1)$ & 2991.486 & 2991.446 \\
$Q_{1}(2)$ & 2987.268 & 2987.230 \\
$Q_{1}(3)$ & 2980.967 & 2980.877 \\
$Q_{1}(4)$ & 2972.597 & 2972.557 \\
$S_{1}(0)$ & 3166.300 & \\
$S_{1}(1)$ & 3278.434 & \\
$S_{1}(2)$ & 3387.220 & \\
$S_{1}(3)$ & 3492.064 & \\
$S_{1}(4)^{\mathrm{a}}$ & 3592.559 & \\
$Q_{2}(0)^{\mathrm{b}}$ & 5868.00 & \\
$Q_{2}(1)^{\mathrm{b}}$ & 5863.84 & \\
$Q_{2}(2)^{\mathrm{b}}$ & 5855.48 & \\
\hline
\end{tabular}

"Not observed in this experiment because water contaminated sample. This is calculated value.

b At 530 psi. 
TABLE $Y$

A Comparison of $\mathrm{H}_{2}$ Spectrum Observed in This Experiment with That Obenzed By Others

\begin{tabular}{cccc}
\hline Line & Obs. in This Exp. & Rank et al. & Stoicheff Raman \\
\hline$Q_{1}(0)$ & 4161.158 & 4161.181 & 4161.134 \\
$Q_{1}(1)$ & 4155.240 & $4155.258_{6}$ & 4155.202 \\
$Q_{1}(2)$ & 4143.449 & $4143.466_{4}$ & 4243.387 \\
$Q_{1}(3)$ & 4125.867 & 4125.871 & 4125.832 \\
$Q_{1}(4)$ & 4102.595 & & \\
$Q_{1}(5)$ & 4073.745 & & \\
$S_{1}(0)$ & 4497.807 & $4497.838_{b}$ & \\
$S_{1}(1)$ & 4712.923 & $4712.906_{6}$ & \\
$S_{1}(2)$ & 4916.970 & $4917.011_{\delta}$ & \\
$S_{1}(3)$ & 5108.386 & $5108.406_{6}$ & \\
$Q_{2}(0)$ & $8086.86^{\mathrm{b}}$ & & \\
$Q_{2}(1)$ & 8075.27 & 8075.314 & \\
$Q_{2}(2)$ & $8051.80^{\mathrm{c}}$ & & \\
\hline
\end{tabular}

: Observed by Herzberg.

b. At 530 psi.

$\therefore$ At 530 psi.

\section{TABLE VI}

The Molecular Constants of Deuterium Determined in This Work Compared to Values Detramined by Others

\begin{tabular}{lcc}
\hline & This Exp. & Stoicheff \\
$B_{0}$ & $29.9063 \pm 0.0005$ & $29.910_{5} \pm 0.005$ \\
$B_{1}$ & $28.8488 \pm 0.0005$ & $28.848_{2}$ \\
$\mathrm{I}_{0}$ & $0.01139_{6} \pm 0.00005$ & $0.0113_{4} \pm 0.0003$ \\
$\mathrm{I}_{1}$ & $0.0109 \overline{5}_{8} \pm 0.00005$ & $0.0107_{5}$ \\
$\mathrm{II}_{0}$ & $7.27 \times 10^{-6} \pm 9 \times 10^{-7}$ & $3.6 \times 10^{-6} \pm 5 \times 10^{-6}$ \\
$\mathrm{I}_{1}$ & $3.5_{6} \times 10^{-6} \pm 8 \times 10^{-7}$ & $3.6 \times 10^{-6}$ \\
$\nu_{11} \rightarrow 1$ & $2993.60_{6} \pm .02$ & $2993.54_{8} \pm .02$ \\
\hline
\end{tabular}

TABLE VII

The Molecular Constants of Hydrogen Determined in This Work Compared to Values Determined by Others

\begin{tabular}{lccc}
\hline & This Exp. & Rank et al. & Stoicheff \\
\hline$B_{0}$ & $59.334_{3} \pm 0.003$ & 59.336 & $59.339_{2} \pm 0.005$ \\
$B_{1}$ & $56.371_{6} \pm 0.003$ & $56.371_{2}$ & $56.377_{8}$ \\
$D_{0}$ & $0.0457_{0} \pm 0.0003$ & $0.0458_{4}$ & $0.0459_{9} \pm 0.003$ \\
$D_{1}$ & $0.0437_{8} \pm 0.0003$ & $0.0438_{1}$ & $0.0443_{5}$ \\
$H_{0}$ & $4.5_{8} \times 10^{-5} \pm 8 \times 10^{-6}$ & $4.9_{0} \times 10^{-5}$ & $5.2 \times 10^{-5} \pm 5 \times 10^{-6}$ \\
$H_{1}$ & $3.59 \times 10^{-5} \pm 8 \times 10^{-4}$ & $3.8_{4} \times 10^{-5}$ & $5.2 \times 10^{-5}$ \\
$\nu_{0} \rightarrow 1$ & $4161.15_{8} \pm 0.02$ & $4161.181_{5}$ & $4161.13_{4} \pm 0.02$ \\
\hline
\end{tabular}


The deuterium hydride constants are within experimental error of Durie and Herzberg's (17) and Stoicheff's values with the exception of $\nu_{0} \rightarrow 1$. Stoicheff's value is about $0.1 \mathrm{~cm}^{-1}$ less than the valuc found by Durie and Herzberg and here. The overtone line $Q_{2}(1)$ found here is in agreement with Rank et al. (5).

TABLE VIII

The Molecular Constants of Deuterium Hydride Determined in This Work Compared to Values Determined by Others

\begin{tabular}{lccc}
\hline & This Exp. & Durie and Herzberg & Sloichefi \\
\hline$B_{0}$ & $44.668 \pm 0.006$ & $44.668_{7}$ & $44.667_{\mathrm{s}} \pm 0.005$ \\
$B_{1}$ & $42.736 \pm 0.006$ & $42.742_{7}$ & $42.741_{1}$ \\
$\mathrm{D}_{0}$ & $0.0259_{6} \pm 0.0006$ & 0.0263 & $0.0259_{2} \pm 0.0005$ \\
$\mathrm{D}_{1}$ & $0.0248_{7} \pm 0.0006$ & $0.0254_{4}$ & $0.0255_{3}$ \\
$\mathrm{H}_{0}$ & $2.21 \times 10^{-5}$ & & $2.2 \times 10^{-5}$ \\
$\mathrm{H}_{1}$ & $1.4 \times 10^{-5}$ & & $2.2 \times 10^{-5}$ \\
$\nu_{0} \rightarrow 1$ & $3632.16_{0} \pm 0.03$ & $3632.14_{9} \pm 0.02$ & $3632.05_{5} \pm 0.02$ \\
\hline
\end{tabular}

'TABLE' IX

Observed Line Widths of $Q_{1}(J)$ Line of $\mathrm{H}_{2}$ AND $\mathrm{D}_{2}$ IN $\mathrm{CM}^{-1}$

\begin{tabular}{ccccccc}
\hline Pressure & \multirow{2}{*}{ Electric Field } & $Q(0)$ & $Q(1)$ & $Q(2)$ & $Q(3)$ & $Q(4)$ \\
\hline & & \multicolumn{2}{c}{ Deuterium } \\
509 & $121500 \mathrm{~V} / \mathrm{cm}$ & 0.264 & 0.208 & 0.234 & 0.234 & 0.234 \\
400 & 121500 & 0.226 & 0.190 & 0.211 & 0.211 & 0.217 \\
250 & 121500 & 0.197 & 0.172 & 0.192 & 0.181 & 0.178 \\
500 & 67500 & 0.237 & 0.189 & 0.219 & 0.224 & 0.217 \\
400 & 67500 & 0.212 & 0.186 & 0.192 & 0.206 & 0.200 \\
250 & 67500 & 0.196 & 0.174 & 0.185 & 0.178 & 0.181 \\
100 & 67500 & 0.175 & 0.165 & 0.167 & 0.164 & 0.167 \\
& & \multicolumn{2}{c}{ Hydrogen } & & & \\
560 & $135000 \mathrm{~V} / \mathrm{cm}$ & 0.167 & 0.196 & 0.171 & 0.201 & \\
456 & 135000 & 0.150 & 0.168 & 0.143 & 0.169 & \\
358 & 135000 & 0.136 & 0.149 & 0.129 & 0.142 & \\
256 & 135000 & 0.122 & 0.121 & 0.111 & 0.119 & \\
560 & 94000 & 0.150 & 0.158 & 0.157 & & \\
456 & 94000 & 0.140 & 0.135 & 0.138 & & \\
358 & 94000 & 0.130 & 0.116 & 0.120 & & \\
256 & 94000 & 0.103 & 0.102 & 0.101 & & \\
156 & 94000 & 0.108 & 0.098 & 0.092 & & \\
560 & $67500 \mathrm{~V} / \mathrm{cm}$ & 0.140 & 0.140 & 0.148 & & \\
456 & 67500 & 0.133 & 0.116 & 0.129 & & \\
358 & 67500 & 0.126 & 0.107 & & & \\
256 & 67500 & & 0.102 & & & \\
156 & 67500 & & 0.093 & & & \\
\hline
\end{tabular}

a Spectral slits $0.160 \mathrm{~cm}^{-1}$.

b Spectral slits $0.085 \mathrm{~cm}^{-1}$. 
TABLE X

Slopes of Line Position vs. Density Curves for Hz and $\mathrm{D}_{2}$ in Units of ( $\mathrm{Cm}^{-1}$ Unit Relative Density) $10^{-3 a}$

\begin{tabular}{|c|c|c|c|c|}
\hline Line & I & II & III & IV \\
\hline$Q_{1}(0)$ & $-1.7 \pm .5$ & $-1.8 \pm .2$ & $-2.35 \pm .17$ & -2.34 \\
\hline$Q_{1}(1)$ & $-1.6 \pm .2$ & $-2.9 \pm .2$ & $-3.14 \pm .15$ & -3.18 \\
\hline$Q_{1}(2)$ & $-1.7 \pm .2$ & $-1.5 \pm .2$ & $-2.07 \pm .16$ & -2.02 \\
\hline$Q_{1}(3)$ & $-1.4 \pm .5$ & $-1.8 \pm .3$ & $-2.25 \pm .17$ & -1.85 \\
\hline$Q_{1}(4)$ & $-2.0 \pm .9$ & $-1.7 \pm .3$ & & \\
\hline$Q_{1}(5)$ & $-1.7 \pm 1.5$ & & & \\
\hline$S_{1}(0)$ & $-0.9 \pm .6$ & $-1.3 \pm .2$ & & -1.43 \\
\hline$S_{1}(1)$ & $-0.6 \pm .4$ & $-1.3 \pm .2$ & & -2.40 \\
\hline$S_{1}(2)$ & $-0.8 \pm .4$ & $-1.8 \pm .3$ & & -3.53 \\
\hline$S_{1}(3)$ & $-1.06 \pm .7$ & $-1.0 \pm .3$ & & -1.77 \\
\hline$O_{1}(2)$ & $-0.8 \pm .7$ & $-2.7 \pm .3$ & & \\
\hline$O_{1}(3)$ & $-0.8 \pm .7$ & $-2.6 \pm .8$ & & \\
\hline a Key: & $\begin{aligned} \mathrm{I} & =\mathrm{S} \\
\mathrm{II} & =\mathrm{S} \\
\mathrm{III} & =\mathrm{S} \\
\mathrm{IV} & =\mathrm{S}\end{aligned}$ & $\begin{array}{l}\text { iven by this } \\
\text { iven by this } \\
\text { iven by May } \\
\text { iven by Ranl }\end{array}$ & $\begin{array}{l}\mathrm{D}_{2} \\
\mathrm{r} \mathrm{H}_{2}\end{array}$ & \\
\hline
\end{tabular}

Table IX shows the observed line widths as a function of electric field and pressure. The $Q_{1}(1)$ line of hydrogen and deuterium are narrower than the others as Van Kranendonk's theory predicts, but Allin et al. (6) and Rank et al. (5) have found that the magnitude of the broadening coefficient differs from the theory. No attempt was made to determine the broadening coefficients here because the masking effect of the slit was too great to give meaningful results except. at the highest pressure.

It is noted in Table IX that the line widths appear to be electric field dependent. Possible broadening mechanism are Stark broadening and induced dipole-quadrupole broadening. The former appears to be ruled out since the $J=0$ line is broadened. The latter is now under theoretical consideration. The electric field dependence of the $Q_{1}(1)$ line of hydrogen is partly due to nonlinear absorption.

The slopes of the line position versus wavelength are listed in Table X. Table X also compares the data of this experiment with that of May et al. (2) and Rank et al. (4), (5).

\section{SUMMARY}

This study of the vibration-rotation, electric field induced spectra of hydrogen has yielded enough data to determine accurate molecular constants. In addition to line positions, it was shown in this experiment that valuable data on pressure broadening and pressure shifts could be obtained by this means. 


\section{ACKNOWLEDGEMENT}

This work was partially supported by Air Force Contract No. AF 19(604)-2071. This work was done at the University of Michigan.

RECEIvED: October 30, 1967

\section{REFERENCES}

1. A. MAY, V. Degen, J. Stryland, and H. Welsh, Can. J. Phys. 39, 1769 (1961).

2. A. May, G. Varghese, J. Struland, and H. Welsh, Can. J. Phys. 42, 1058 (1964).

3. D. H. Rank and T. A. Wiggins, J. Opt. Soc. Am. 53, 759 (1963).

4. U. Fink, T. A. Wiggins, AND D. H. Rank, J. Mol. Spectry. 18, 384 (1965).

5. J. V. Foltz, D. H. Rank, and T. A. Wiggins, J. Mol. Spectry. 21, 203 (1966).

6. E. Aluin, A. May, B. Stoicheff, J. Stryland, and H. Welsh, Appl. Optics. 6, 1597 (1967).

7. J. Fuutak and J. Van Kranendonk, Can. J. Physics, 40, 1085 (1962); ibid. 41, 21 (1963).

8. J. Van Kranendonk, Can. I. Phys. 41, 433 (1963).

9. E. U. Condon, Phys. Rev. 41, 759 (1932).

10. M. F. Crawford and I. Dagg, Phys. Rev. 91, 1564 (1953).

11. M. Crawford and R. MacDonald, Can. J. Phys. 36, 1022 (1958).

12. R. W. Terhune and C. W. Peters, J. Mol. Spectry. 3, 138 (1959).

13. H. W. MarshalL, JR. A Multipass Vacuum Grating Ebert Spectrometer. Unpublished

Doctoral dissertation, University of Michigan (1961).

14. R. S. Mautner and O. H. Schade, RCA Rev. 8, 43 (1947).

15. J. L. Dunham, Phys. Rev. 4, 721 (1932).

16. B. P. Stoicheff, Can. J. Phys. 35, 730 (1957).

17. R. Durie and G. Herzberg, Can. J. Phys. 38, 806 (1960). 